\title{
LIMIT THEOREMS FOR MOVING AVERAGES WITH RANDOM COEFFICIENTS AND HEAVY-TAILED NOISE
}

\author{
RAFAŁ KULIK, ${ }^{*}$ University of Wroctaw and University of Ottawa
}

\begin{abstract}
We consider a stationary moving average process with random coefficients, $X_{t}=$ $\sum_{k=0}^{\infty} C_{t, k} Z_{t-k}$, generated by an array, $\left\{C_{t, k}, t \in \mathbb{Z}, k \geq 0\right\}$, of random variables and a heavy-tailed sequence, $\left\{Z_{t}, t \in \mathbb{Z}\right\}$. We analyze the limit behavior using a point process analysis. As applications of our results we compare the limiting behavior of the moving average process with random coefficients with that of a standard $\mathrm{MA}(\infty)$ process.
\end{abstract}

Keywords: Nonlinear process; point process; self-normalized sum

2000 Mathematics Subject Classification: Primary 62M10

Secondary 60E07; 60G10; 60G55

\section{Introduction}

Many data sets from telecommunications, finance, and economics exhibit heavy tails. A key question is how to fit models to such data. In the setting of a stationary time series with finite variance, autoregressive moving average (MA) processes are sufficient for data analysis, and we may use standard techniques to fit an appropriate one. If the variance is infinite and a data sequence, $\left\{X_{t}, t \in \mathbb{Z}\right\}$, is generated by the linear process

$$
X_{t}=\sum_{k=0}^{\infty} c_{k} Z_{t-k},
$$

where $\left\{Z_{t}, t \in \mathbb{Z}\right\}$ is a sequence of independent and identically distributed (i.i.d.) regularly varying random variables, then we may use the sample autocorrelation function to fit an appropriate model; see, e.g. [6]. However, when there are nonlinearities, the sample autocorrelation function need not be consistent. In particular, it may converge in distribution to a nondegenerate random variable. This can result in an inappropriate model being selected. This phenomenon was observed in [8] for bilinear processes

$$
X_{t}=c X_{t-1} Z_{t-1}+Z_{t}
$$

where $|c|^{\alpha / 2} \mathrm{E}\left[Z_{1}^{\alpha / 2}\right]<1, \alpha>0$, and $\left\{Z_{t}, t \in \mathbb{Z}\right\}$ are i.i.d. and regularly varying. Also, if the model is linear then $X$, a generic random variable of the sequence $\left\{X_{t}, t \in \mathbb{Z}\right\}$, has a regularly varying tail with index $\alpha$ if and only if $Z$ also does. On the other hand, if

$$
X_{t}=Y_{t} X_{t-1}+Z_{t}, \quad t \in \mathbb{Z},
$$

where $\left\{Y_{t}, t \in \mathbb{Z}\right\}$ are i.i.d. but not necessarily independent of $\left\{Z_{t}, t \in \mathbb{Z}\right\}$, then regular variation may appear even though the noise variables $Z_{t}$ all have finite moments; see, e.g. [10]. We refer

Received 22 October 2004; revision received 13 September 2005.

* Postal address: Department of Mathematics and Statistics, University of Ottawa, 585 King Edward Avenue, Ottawa, ON, K1N 6N5, Canada. Email address: rkuli438@ science.uottawa.ca 
the reader to [9, Chapter 7] as well as [12] and [14] for further discussion of nonlinear time series.

Therefore, in the infinite-variance case, new limit theorems and statistical tests for detecting possible nonlinearities are required. To obtain limit theorems for the models mentioned above, we observe that each of them can be written as a moving average with random coefficients. To be more specific, assume that $\left\{Z_{t}, t \in \mathbb{Z}\right\}$ is a sequence and that $\mathcal{C}=\left\{C_{t, k}, t \in \mathbb{Z}, k \geq 0\right\}$ is an infinite array of random variables. Then

$$
X_{t}=\sum_{k=0}^{\infty} C_{t, k} Z_{t-k}, \quad t \in \mathbb{Z},
$$

is the moving average process with random coefficients.

Now, if the sequence $\left\{\left(Y_{t}, Z_{t}\right), t \in \mathbb{Z}\right\}$ is i.i.d. then model (1.3) may be represented as (1.4) by taking $C_{t, k}=\prod_{i=0}^{k-1} Y_{t-i}$. Thus, $C_{t+k, k}=Y_{t+k} \cdots Y_{t+1}$ and, hence, for fixed $t, Z_{t}$ is independent of $\left\{C_{t+k, k}, k \geq 0\right\}$. Also, for the bilinear model (1.2) with i.i.d. noise sequence $\left\{Z_{t}, t \in \mathbb{Z}\right\}$, we have $C_{t, k}=c^{k} \prod_{i=1}^{k-1} Z_{t-i}$ and $Z_{t-k}$ is replaced with $Z_{t-k}^{2}$ in (1.4). Consequently, $C_{t+k, k}=c^{k} Z_{t+k-1} \cdots Z_{t+1}$ is independent of $Z_{t}$ for all $k \geq 0$.

For random variables, $X_{t}$, defined by (1.4), our main goal is to obtain point process limits for

$$
\sum_{t=1}^{n} \delta_{a_{n}^{-1} X_{t}}, \quad \sum_{t=1}^{n} \delta_{a_{n}^{-1}\left(X_{t}, \ldots, X_{t-h}\right)},
$$

where $\delta$ is the Dirac measure and $\left(a_{n}\right)$ an appropriate normalizing sequence. In order to do this we shall first prove that the tail of $X_{t}$ behaves as if we have considered the standard $\mathrm{MA}(\infty)$ model (1.1) with coefficients $c_{k}=\left\|C_{t, k}\right\|_{\alpha}:=\mathrm{E}\left[\left|C_{t, k}\right|^{\alpha}\right]^{1 / \alpha}$. Hence, in the onedimensional case we generalize the result of [16] and, as a corollary, obtain the tail asymptotics for bilinear processes [8] or stochastic recurrences [11]. We also obtain the tail asymptotics for $S_{n}=\sum_{t=1}^{n} X_{t}$, for fixed $n$, generalizing a result of [11]. Note that in this case the tail asymptotics for $S_{n}$ does not agree with the $\operatorname{MA}(\infty)$ process, unless $\alpha=1$.

Having established the tail asymptotics we may proceed with point process limits. For this it is reasonable to expect that we need stronger assumptions on the dependence structure. We assume that the array $\mathcal{C}$ is independent of $\left\{Z_{t}, t \in \mathbb{Z}\right\}$. We note that, for the stochastic recurrence model, Konstantinides and Mikosch [11] obtained the point process limit without such an independence assumption. Their approach uses the theory developed in [4] and [5]. However, in the general moving average model (1.4), the conditions of Theorem 2.2 of [5] are rather difficult to verify. By viewing the stochastic recurrence model as a particular case of the moving average process with random coefficients, we may apply well-known techniques for linear processes to provide a unified tool for several types of time series. The point process limit results allow us to point out some differences between the limiting behaviors of the $\operatorname{MA}(\infty)$ model and the general moving average process (1.4). Also, we obtain a simple rule to check whether or not the stochastic recurrence model (1.3) is linear (i.e. has constant, deterministic $Y_{t}$ ).

The paper is organized as follows. In Section 2 we deal with the tail asymptotics of $X_{t}$ and $S_{n}$. In particular, we apply our results to bilinear processes and stochastic recurrences with heavy-tailed noise. In Section 3 we obtain the point process limits. Those results are applied in Section 4 to the asymptotic behavior of various statistics. 


\section{Tail asymptotics}

In the sequel we write $f(x) \sim g(x)$ if $\lim _{x \rightarrow \infty} f(x) / g(x)=1$ and $f(x)=o(g(x))$ if $\lim _{x \rightarrow \infty} f(x) / g(x)=0$. Moreover, we shall denote by $A$ a generic random variable of any stationary sequence $\left\{A_{t}, t \in \mathbb{Z}\right\}$.

We assume that the following conditions hold.

(A) The sequence $\left\{Z_{t}, t \in \mathbb{Z}\right\}$ is i.i.d.

(B) $Z_{t}, t \in \mathbb{Z}$, are heavy tailed; i.e. $\mathrm{P}(|Z|>x)=x^{-\alpha} L(x)$, where $\alpha>0, L$ is slowly varying at infinity, and, for $p+q=1, p, q \geq 0$, we have

$$
\lim _{x \rightarrow \infty} \frac{\mathrm{P}(Z>x)}{\mathrm{P}(|Z|>x)}=p, \quad \lim _{x \rightarrow \infty} \frac{\mathrm{P}(Z<-x)}{\mathrm{P}(|Z|>x)}=q .
$$

Recall that this is equivalent to the vague convergence

$$
n \mathrm{P}\left(a_{n}^{-1} Z \in \cdot\right) \rightarrow v(\cdot),
$$

where $v$ has density $v(\mathrm{~d} x)=\alpha x^{-\alpha-1} \mathbf{1}_{(0, \infty]}(x)+q p^{-1} \alpha(-x)^{-\alpha-1} \mathbf{1}_{[-\infty, 0)}(x)$ and $\left(a_{n}\right)$ is an increasing sequence such that $\mathrm{P}\left(|Z|>a_{n}\right) \sim n^{-1}$. Here $\mathbf{1}_{A}(\cdot)$ denotes the indicator function of the set (or event) $A$.

(C) For all fixed $t \in \mathbb{Z}, Z_{t}$ is independent of $\left\{C_{t+k, k}, k \geq 0\right\}$. In particular, for fixed $t \in \mathbb{Z}$ and $k \geq 0$, the random variables $C_{t, k}$ and $Z_{t-k}$ are independent.

(D) For each $t$, if $\alpha<1$ then there exists a $\delta, 0<\delta<\alpha$, such that $\alpha+\delta<1$ and

$$
\sum_{k=0}^{\infty} \mathrm{E}\left[\left|C_{t, k}\right|^{\alpha+\delta}\right]<\infty, \quad \sum_{k=0}^{\infty} \mathrm{E}\left[\left|C_{t, k}\right|^{\alpha-\delta}\right]<\infty .
$$

If $\alpha>1$ then there exists a $\delta>0$ such that

$$
\sum_{k=0}^{\infty} \mathrm{E}\left[\left|C_{t, k}\right|^{\alpha+\delta}\right]^{1 /(\alpha+\delta)}<\infty, \quad \sum_{k=0}^{\infty} \mathrm{E}\left[\left|C_{t, k}\right|^{\alpha-\delta}\right]^{1 /(\alpha+\delta)}<\infty .
$$

Conditions (A)-(D) imply that the infinite series $\sum_{k=0}^{\infty} C_{t, k} Z_{t-k}$ converges almost surely and, hence, that $\left\{X_{t}, t \in \mathbb{Z}\right\}$ is well defined; see, e.g. [16]. Clearly, from the above we also find that, for all $t$ and $k$ and some $\delta>0$,

$$
\mathrm{E}\left[\left|C_{t, k}\right|^{\alpha+\delta}\right]<\infty .
$$

Assume that $t \in \mathbb{Z}$ is fixed but arbitrary. The main result of this section is as follows.

Proposition 2.1. Assume that conditions (A)-(D) hold. Then

$$
\lim _{x \rightarrow \infty} \frac{\mathrm{P}\left(X_{t}>x\right)}{\mathrm{P}(|Z|>x)}=\sum_{k=0}^{\infty}\left(p \mathrm{E}\left[\left|C_{t, k}\right|^{\alpha} \mathbf{1}_{\left\{C_{t, k}>0\right\}}\right]+q \mathrm{E}\left[\left|C_{t, k}\right|^{\alpha} \mathbf{1}_{\left\{C_{t, k}<0\right\}}\right]\right) .
$$

To prove Proposition 2.1 we begin with a series of lemmas. First, by a straightforward extension of [2] we find that

$$
\mathrm{P}(Z Y>x) \sim\left(p \mathrm{E}\left[Y^{\alpha} \mathbf{1}_{\{Y>0\}}\right]+q \mathrm{E}\left[Y^{\alpha} \mathbf{1}_{\{Y<0\}}\right]\right) \mathrm{P}(|Z|>x)
$$

for two independent random variables, $Z$ and $Y$, such that (B) holds for $Z$ and $\mathrm{E}\left[|Y|^{\alpha+\delta}\right]<\infty$ for some $\delta>0$. 
Lemma 2.1. Let $U_{k}, k=0, \ldots, m$, be random variables with $\mathrm{P}\left(\left|U_{0}\right|>x\right)=x^{-\alpha} L(x)$. If, for $k=0, \ldots, m$,

$$
\lim _{x \rightarrow \infty} \frac{\mathrm{P}\left(U_{k}>x\right)}{\mathrm{P}\left(\left|U_{0}\right|>x\right)}=c_{k}
$$

and

$$
\lim _{x \rightarrow \infty} \frac{\mathrm{P}\left(U_{k}>x, U_{l}>x\right)}{\mathrm{P}\left(\left|U_{0}\right|>x\right)}=0, \quad k \neq l,
$$

then

$$
\lim _{x \rightarrow \infty} \frac{\mathrm{P}\left(\sum_{k=0}^{m} U_{k}>x\right)}{\mathrm{P}\left(\left|U_{0}\right|>x\right)}=\sum_{k=0}^{m} c_{k}
$$

Proof. The lower bound follows from

$$
\lim _{x \rightarrow \infty} \frac{\mathrm{P}\left(\sum_{k=0}^{m} U_{k}>x\right)}{\mathrm{P}\left(\left|U_{0}\right|>x\right)} \geq \lim _{x \rightarrow \infty} \frac{\mathrm{P}\left(\sum_{k=0}^{m} U_{k} \mathbf{1}_{\left\{U_{k}>0\right\}}>x\right)}{\mathrm{P}\left(\left|U_{0}\right|>x\right)}
$$

and Lemma 2.1 of [8]. The upper bound may be obtained by the same calculation as in the proof of Lemma A3.26 of [9], using (2.2) instead of independence.

Lemma 2.2. ([16].) Assume that $Z$ is nonnegative and that $\mathrm{P}(Z>x)=x^{-\alpha} L(x), \alpha>0$. Then there exists an $x_{0}$ such that

$$
\mathrm{E}\left[(c Z \wedge x)^{\alpha+\eta}\right] \leq \begin{cases}\text { const. } c^{\alpha+\eta} x^{\alpha+\eta} \mathrm{P}(Z>x) & \text { if } c \geq 1 \text { and } x / c \geq x_{0}, \\ \text { const. } c^{\alpha-\eta} x^{\alpha-\eta} \mathrm{P}(Z>x) & \text { if } c<1 \text { and } x>x_{0} .\end{cases}
$$

Lemma 2.3. Assume that (A), (B), and (2.1) hold. Then, for all $t$ and $k \neq l$,

$$
\lim _{x \rightarrow \infty} \frac{\mathrm{P}\left(C_{t, k} Z_{t-k}>x, C_{t, l} Z_{t-l}>x\right)}{\mathrm{P}(|Z|>x)}=0 .
$$

Proof. Since $\mathrm{P}\left(C_{t, k} Z_{t-k}>x, C_{t, l} Z_{t-l}>x\right) \leq \mathrm{P}\left(\left|C_{t, k} Z_{t-k}\right|>x,\left|C_{t, l} Z_{t-l}\right|>x\right)$ we may assume without loss of generality that the random variables are nonnegative. Let $\varepsilon>0$ and choose $k \neq l$. We have

$$
\begin{aligned}
\mathrm{P}\left(C_{t, k} Z_{t-k}>x, C_{t, l} Z_{t-l}>x\right) \leq & \mathrm{P}\left(C_{t, k} \mathbf{1}_{\left\{C_{t, k}>\varepsilon\right\}} Z_{t-k}>x\right)+\mathrm{P}\left(C_{t, l} \mathbf{1}_{\left\{C_{t, l}>\varepsilon\right\}} Z_{t-l}>x\right) \\
& +\mathrm{P}\left(C_{t, k} \leq \varepsilon, C_{t, l} \leq \varepsilon, C_{t, k} Z_{t-k}>x, C_{t, l} Z_{t-l}>x\right) \\
\leq & \mathrm{P}\left(C_{t, k} \mathbf{1}_{\left\{C_{t, k}>\varepsilon\right\}} Z_{t-k}>x\right)+\mathrm{P}\left(C_{t, l} \mathbf{1}_{\left\{C_{t, l}>\varepsilon\right\}} Z_{t-l}>x\right) \\
& +\mathrm{P}\left(Z_{t-k}>x / \varepsilon, Z_{t-l}>x / \varepsilon\right) .
\end{aligned}
$$

For the first two terms, respectively $I_{1}$ and $I_{2}$, by (2.1) and Breiman's lemma we have

$$
\lim _{x \rightarrow \infty} \frac{I_{1}}{\mathrm{P}(Z>x)}=\mathrm{E}\left[C_{t, k}^{\alpha} \mathbf{1}_{\left\{C_{t, k}>\varepsilon\right\}}\right],
$$

which converges to 0 as $\varepsilon \rightarrow \infty$, since

$$
\limsup _{\varepsilon \rightarrow \infty} \mathrm{E}\left[C_{t, k}^{\alpha} \mathbf{1}_{\left\{C_{t, k}>\varepsilon\right\}}\right] \leq \mathrm{E}\left[\limsup _{\varepsilon \rightarrow \infty} C_{t, k}^{\alpha} \mathbf{1}_{\left\{C_{t, k}>\varepsilon\right\}}\right] .
$$

Moreover, the third term is $o(\mathrm{P}(Z>x))$, by (A). 
Proof of Proposition 2.1. For all finite $m \geq 1$, we have

$$
\begin{aligned}
\mathrm{P}\left(X_{t}>x\right) \geq & \mathrm{P}\left(\sum_{k=0}^{m} C_{t, k} \mathbf{1}_{\left\{C_{t, k}>0\right\}} Z_{t-k} \mathbf{1}_{\left\{Z_{t-k}>0\right\}}>x\right) \\
& +\mathrm{P}\left(\sum_{k=0}^{m} C_{t, k} \mathbf{1}_{\left\{C_{t, k}<0\right\}} Z_{t-k} \mathbf{1}_{\left\{Z_{t-k}<0\right\}}>x\right) .
\end{aligned}
$$

Thus, the lower bound follows from Lemmas 2.1 and 2.3 and the standard argument; see, e.g. [13, pp. 228-230].

To determine the upper bound we use an approach similar to that of [16]. We have

$$
\begin{aligned}
\lim _{x \rightarrow \infty} & \frac{\mathrm{P}\left(\sum_{k=0}^{\infty} C_{t, k} Z_{t-k}>x\right)}{\mathrm{P}(|Z|>x)} \\
\leq & \lim _{x \rightarrow \infty} \frac{\mathrm{P}\left(\sum_{k=0}^{m} C_{t, k} Z_{t-k}>(1-\varepsilon) x\right)}{\mathrm{P}(|Z|>x)}+\lim _{x \rightarrow \infty} \frac{\sum_{k=m+1}^{\infty} \mathrm{P}\left(\left|C_{t, k} Z_{t-k}\right|>\varepsilon x\right)}{\mathrm{P}(|Z|>x)} \\
& +\lim _{x \rightarrow \infty} \frac{\mathrm{P}\left(\sum_{k=m+1}^{\infty}\left|C_{t, k} Z_{t-k}\right| \mathbf{1}_{\left\{\left|C_{t, k} Z_{t-k}\right|<\varepsilon x\right\}}>\varepsilon x\right)}{\mathrm{P}(|Z|>x)} \\
= & : I_{1}+I_{2}+I_{3} .
\end{aligned}
$$

The first term, $I_{1}$, gives the required asymptotics. By Breiman's lemma and (D), $I_{2} \rightarrow 0$ provided that we can interchange limits with summations. To see this, consider that

$$
\begin{aligned}
& \frac{\mathrm{P}\left(\left|C_{t, k} Z_{t-k}\right|>\varepsilon x\right)}{\mathrm{P}(|Z|>x)} \\
& \quad=\frac{\mathrm{E}\left[\mathbf{1}_{\left\{\left|C_{t, k}\right| \leq \varepsilon\right\}} \mathrm{P}\left(\left|C_{t, k} Z_{t-k}\right|>\varepsilon x \mid C_{t, k}\right)\right]}{\mathrm{P}(|Z|>x)}+\frac{\mathrm{E}\left[\mathbf{1}_{\left\{\left|C_{t, k}\right|>\varepsilon\right\}} \mathrm{P}\left(\left|C_{t, k} Z_{t-k}\right|>\varepsilon x \mid C_{t, k}\right)\right]}{\mathrm{P}(|Z|>x)} \\
& \quad \leq \text { const. }\left(\mathrm{E}\left[\mathbf{1}_{\left\{\left|C_{t, k}\right| \leq \varepsilon\right\}}\left|C_{t, k}\right|^{\alpha+\delta}\right]+\mathrm{E}\left[\mathbf{1}_{\left\{\left|C_{t, k}\right|>\varepsilon\right\}}\left|C_{t, k}\right|^{\alpha-\delta}\right]\right) \\
& \quad \leq \text { const. }\left(\mathrm{E}\left[\left|C_{t, k}\right|^{\alpha+\delta}\right]+\mathrm{E}\left[\left|C_{t, k}\right|^{\alpha-\delta}\right]\right),
\end{aligned}
$$

which bound is summable, by (D).

It remains to show that $I_{3}=o(1)$. We may assume that the random variables are nonnegative. First, note that

$$
C_{t, k} Z_{t-k} \mathbf{1}_{\left\{C_{t, k} Z_{t-k}<\varepsilon x\right\}} \leq C_{t, k} Z_{t-k} \wedge \varepsilon x .
$$

Using Lemma 2.2, for $\alpha \in(0,1)$ we have

$$
\begin{aligned}
& \mathrm{P}\left(\sum_{k=m+1}^{\infty} C_{t, k} Z_{t-k} \mathbf{1}_{\left\{C_{t, k} Z_{t-k}<\varepsilon x\right\}}>\varepsilon x\right) \\
& \leq \frac{\text { const. }}{x^{\alpha+\eta}}\left\{\sum_{k=m+1}^{\infty} \mathrm{E}\left[\mathrm{E}\left[\left(C_{t, k} Z_{t-k} \wedge \varepsilon x\right)^{\alpha+\eta} \mathbf{1}_{\left\{C_{t, k} \geq \varepsilon\right\}} \mid C_{t, k}\right]\right]\right. \\
& \left.+\mathrm{E}\left[\mathrm{E}\left[\left(C_{t, k} Z_{t-k} \wedge \varepsilon x\right)^{\alpha+\eta} \mathbf{1}_{\left\{C_{t, k}<\varepsilon\right\}} \mid C_{t, k}\right]\right]\right\} \\
& \leq \frac{\text { const. }}{x^{\alpha+\eta}}\left\{\sum_{k=m+1}^{\infty} \mathrm{E}\left[C_{t, k}^{\alpha+\eta}\right]+\sum_{k=m+1}^{\infty} \mathrm{E}\left[C_{t, k}^{\alpha-\eta}\right]\right\}
\end{aligned}
$$


and, hence, $I_{3}=o(1)$ follows by dividing the latter expression by $\mathrm{P}(Z>x)$ and applying condition (D). Similarly, by using Minkowski's inequality, for $\alpha \geq 1$ we have

$$
\begin{aligned}
& \mathrm{P}\left(\sum_{k=m+1}^{\infty} C_{t, k} Z_{t-k} \mathbf{1}_{\left\{C_{t, k} Z_{t-k}<\varepsilon x\right\}}>\varepsilon x\right) \\
& \quad \leq \frac{\text { const. }}{x^{\alpha+\eta}} \mathrm{E}\left[\left(\sum_{k=m+1}^{\infty} C_{t, k} Z_{t-k} \wedge \varepsilon x\right)^{\alpha+\eta}\right] \\
& \quad \leq \frac{\text { const. }}{x^{\alpha+\eta}}\left(\sum_{k=m+1}^{\infty} \mathrm{E}\left[\left(C_{t, k} Z_{t-k} \wedge \varepsilon x\right)^{\alpha+\eta}\right]^{1 /(\alpha+\eta)}\right)^{\alpha+\eta} \\
& \quad \leq \frac{\text { const. }}{x^{\alpha+\eta}}\left(\sum_{k=m+1}^{\infty} \mathrm{E}\left[C_{t, k}^{\alpha+\eta}\right]^{1 /(\alpha+\eta)}+\sum_{k=m+1}^{\infty} \mathrm{E}\left[C_{t, k}^{\alpha-\eta}\right]^{1 /(\alpha+\eta)}\right)^{\alpha+\eta}
\end{aligned}
$$

and condition (D) can again be used to show that $I_{3}=o(1)$. This concludes the proof.

To simplify our notation we shall assume, in the remaining part of this section, that all random variables are nonnegative. Thus, in (B), $p=1$.

Let $S_{n}=\sum_{t=1}^{n} X_{t}$ and note that it may be written as

$$
S_{n}=\sum_{k=0}^{-\infty}\left(\sum_{j=1}^{n} C_{j, j-k}\right) Z_{k}+\sum_{k=1}^{n}\left(\sum_{j=k}^{n} C_{j, j-k}\right) Z_{k} .
$$

By using the same argument as in Proposition 2.1, we obtain the following result.

Proposition 2.2. Assume that conditions $(A)-(D)$ hold. Then

$$
\lim _{x \rightarrow \infty} \frac{\mathrm{P}\left(S_{n}>x\right)}{\mathrm{P}(Z>x)}=\sum_{k=0}^{\infty} \mathrm{E}\left[\left(\sum_{j=1}^{n} C_{j, j+k}\right)^{\alpha}\right]+\sum_{k=1}^{n} \mathrm{E}\left[\left(\sum_{j=k}^{n} C_{j, j-k}\right)^{\alpha}\right] .
$$

Now we apply Propositions 2.1 and 2.2 to some special models.

Example 2.1. The result of Proposition 2.1 means that, in the case of heavy-tailed variables $Z_{t}$ and appropriately chosen $C_{t, k}$, the tail asymptotics of $X$ is the same as it would be if the $C_{t, k}$ were replaced with their $\alpha$-norms, $c_{k}:=\left\|C_{t, k}\right\|_{\alpha}=\mathrm{E}\left[\left|C_{t, k}\right|^{\alpha}\right]^{1 / \alpha}$. If this is the case then condition (D) is equivalent to $\sum_{k=0}^{\infty} c_{k}^{\gamma}<\infty$ for some $\gamma, 0<\gamma<\alpha \wedge 1$, and is standard in the $\mathrm{MA}(\infty)$ case. The tail of the linear process (1.1) can be found in [13, p. 227].

Moreover, by replacing the $C_{t, k}$ in (2.3) with the corresponding $\mathrm{E}\left[C_{k}^{\alpha}\right]^{1 / \alpha}$, we obtain

$$
\lim _{x \rightarrow \infty} \frac{\mathrm{P}\left(S_{n}>x\right)}{\mathrm{P}(Z>x)}=\sum_{k=0}^{\infty}\left(\sum_{j=1}^{n} \mathrm{E}\left[C_{j, j+k}^{\alpha}\right]^{1 / \alpha}\right)^{\alpha}+\sum_{k=1}^{n}\left(\sum_{j=k}^{n} \mathrm{E}\left[C_{j, j-k}^{\alpha}\right]^{1 / \alpha}\right)^{\alpha},
$$

that is, the tail asymptotics for the $\mathrm{MA}(\infty)$ model. Note that (2.4) does not coincide with (2.3) unless $\alpha=1$. Hence, in general, the tail of the partial sum in the random coefficient model is not the same as that in the $\operatorname{MA}(\infty)$ process. Moreover, if $\alpha>1$ then, by Minkowski's inequality, $\mathrm{E}\left[\left(\sum_{j=1}^{n} C_{j}\right)^{\alpha}\right] \leq\left(\sum_{j=1}^{n} \mathrm{E}\left[C_{j}^{\alpha}\right]^{1 / \alpha}\right)^{\alpha}$, and the tail of $S_{n}$ in the random coefficient model is asymptotically dominated by the one in the $\operatorname{MA}(\infty)$ model. 
Example 2.2. Consider the stochastic recurrence equation (1.3), where $\left\{\left(Y_{t}, Z_{t}\right), t \in \mathbb{Z}\right\}$ is an i.i.d. sequence. If $-\infty \leq \mathrm{E}\left[\log \left(Y_{t}\right)\right]<0$ and $\mathrm{E}\left[\log ^{+} Z_{t}\right]<\infty$, then the unique stationary solution to (1.3), $\left\{X_{t}, t \in \mathbb{Z}\right\}$, exists and is given by

$$
X_{t}=Z_{t}+\sum_{k=1}^{\infty} Z_{t-k} \prod_{i=0}^{k-1} Y_{t-i}=\sum_{k=0}^{\infty} Z_{t-k} \prod_{i=0}^{k-1} Y_{t-i}
$$

(we use the convention that $\prod_{i=0}^{-1} \equiv 1$ ). Therefore, this is the special case of (1.4) with $C_{t, k}=\prod_{i=0}^{k-1} Y_{t-i}$.

Assume that (B) holds for $Z$ and that $\mathrm{E}\left[Y^{\alpha}\right]<1$ and $\mathrm{E}\left[Y^{\alpha+\delta}\right]<\infty$ for some $\delta>0$. Then the above series exists and is well defined; see, e.g. [1]. Moreover, there exists some $\eta, 0<\eta<\delta$, such that $\mathrm{E}\left[Y^{\alpha+\eta}\right]<1$; hence, condition (D) is easily verified to hold. Condition (C) is also fulfilled. Therefore, we obtain the following corollary.

Corollary 2.1. ([11].) Assume that (B) holds and that $\mathrm{E}\left[Y^{\alpha+\delta}\right]<\infty$ for some $\delta>0$. If $\mathrm{E}\left[Y^{\alpha}\right]<1$ then

$$
\lim _{x \rightarrow \infty} \frac{\mathrm{P}\left(X_{t}>x\right)}{\mathrm{P}(Z>x)}=\frac{1}{1-\mathrm{E}\left[Y^{\alpha}\right]} .
$$

Moreover, since $C_{j}=Y_{j-1} C_{j-1}$, the first term on the right-hand side of (2.3) is

$$
\sum_{k=0}^{\infty} \mathrm{E}\left[\left(\sum_{j=1}^{n} C_{j+k}\right)^{\alpha}\right]=\mathrm{E}\left[\sum_{i=0}^{n-1} \prod_{j=0}^{i} Y_{j}\right] \sum_{k=0}^{\infty} \mathrm{E}\left[C_{t, k}^{\alpha}\right],
$$

and the second term is

$$
\sum_{k=1}^{n} \mathrm{E}\left[\left(\sum_{j=0}^{n-k} \prod_{i=0}^{j-1} Y_{i}\right)^{\alpha}\right]
$$

we obtain the result of Proposition 3.2 of [11].

Example 2.3. Assume $\left\{Z_{t}, t \in \mathbb{Z}\right\}$ to be an i.i.d. sequence. The tail asymptotics of the bilinear process may be obtained using our framework. Assuming that (B) holds, that $Z^{2}$ is regularly varying with index $\alpha / 2$, and that $c$ is chosen in such a way that $c^{\alpha / 2} \mathrm{E}\left[Z^{\alpha / 2}\right]<1$, the unique stationary solution is given by

$$
X_{t}=Z_{t}+\sum_{k=1}^{\infty} c^{k}\left(\prod_{i=1}^{k-1} Z_{t-i}\right) Z_{t-k}^{2}
$$

Hence, $C_{t, k}=c^{k} \prod_{i=1}^{k-1} Z_{t-i}$ and $Z_{t-k}$ is replaced with $Z_{t-k}^{2}$ in (1.4). Clearly, condition (C) is satisfied, and we hence obtain the following corollary.

Corollary 2.2. ([8].) Assume that (B) holds and that $c^{\alpha / 2} \mathrm{E}\left[Z^{\alpha / 2}\right]<1$. Then

$$
\lim _{x \rightarrow \infty} \frac{\mathrm{P}\left(X_{t}>x\right)}{\mathrm{P}\left(Z^{2}>x\right)}=\frac{c^{\alpha / 2}}{1-c^{\alpha / 2} \mathrm{E}\left[Z^{\alpha / 2}\right]} .
$$

Moreover, (2.3) is also valid. 


\section{Point process limit}

Let $E$ be a locally compact Hausdorff topological space and let $M_{\mathrm{p}}(E)$ be a space of Radon point measures on $E$. The space $M_{\mathrm{p}}(E)$ is equipped with vague metric $d(\cdot, \cdot)$. We say that a sequence of measures, $\mu_{n} \in M_{\mathrm{p}}(E)$, converges vaguely to $\mu \in M_{\mathrm{p}}(E)$ if $\int_{E} f \mathrm{~d} \mu_{n} \rightarrow \int_{E} f \mathrm{~d} \mu$ for all positive continuous functions on $E$ with compact support. The space of all nonnegative continuous functions on $E$ with compact support will be denoted by $C_{K}^{+}(E)$.

Throughout this section we shall assume that, in addition to (A), (B), and (D), the following conditions hold.

$\left(\mathrm{C}^{\prime}\right)$ The array $\left\{C_{t, k}, t \in \mathbb{Z}, k \geq 0\right\}$ is independent of $\left\{Z_{t}, t \in \mathbb{Z}\right\}$.

(E) For each fixed $m$, the sequence $\left\{\left(C_{t, 0}, \ldots, C_{t, m}\right), t \in \mathbb{Z}\right\}$ is strongly mixing.

Assume that the $\mathbb{R}^{\infty}$-valued random elements $\boldsymbol{C}_{t}=\left\{C_{t, k}, k \geq 0\right\}$ form the stationary sequence $\left\{\boldsymbol{C}_{t}, t \geq 1\right\}$. By $\left(\mathrm{C}^{\prime}\right)$, the sequence $\left\{X_{t}, t \geq 1\right\}$ is stationary. Also, suppose that the $\mathbb{R}^{\infty}$-valued random elements $V_{t}=\left(V_{t, 0}, V_{t, 1}, \ldots\right), t \in \mathbb{Z}$, are i.i.d. with the same distribution as $\boldsymbol{C}_{0}$.

A Poisson process on $E$ with mean measure $v$ will be denoted by $\operatorname{PRM}(v)$. It is known that, for the linear process (1.1),

$$
N_{n}:=\sum_{t=1}^{\infty} \delta_{a_{n}^{-1} X_{t}} \stackrel{\mathrm{w}}{\rightarrow} \sum_{t=1}^{\infty} \sum_{k=0}^{\infty} \delta_{c_{k} j_{t}}
$$

in $M_{\mathrm{p}}(E)$, where $\stackrel{\mathrm{W}}{\rightarrow}$ ' denotes weak convergence and $\sum_{t=1}^{\infty} \delta_{j_{t}}$ is a $\operatorname{PRM}(v)$ with density

$$
v(\mathrm{~d} x)=\alpha x^{-\alpha-1} \mathbf{1}_{(0, \infty]}(x)+q p^{-1} \alpha(-x)^{-\alpha-1} \mathbf{1}_{[-\infty, 0)}(x)
$$

(see [13, Proposition 4.27]). In the general model (1.4) we have the following results.

Theorem 3.1. Suppose that the stationary sequence $\left\{X_{t}\right\}$ is given by (1.4). Assume that $(A)$, $(B),\left(C^{\prime}\right),(D)$, and $(E)$ hold. Then

$$
\sum_{t=1}^{n} \delta_{a_{n}^{-1} X_{t}} \stackrel{\mathrm{w}}{\rightarrow} \sum_{t=1}^{\infty} \sum_{k=0}^{\infty} \delta_{j_{t}} V_{t, k}
$$

in $M_{\mathrm{p}}([-\infty, \infty] \backslash\{0\})$.

Theorem 3.2. Under the assumptions of Theorem 3.1 we have

$$
\sum_{t=1}^{n} \delta_{a_{n}^{-1}\left(X_{t}, \ldots, X_{t-h}\right)} \stackrel{\mathrm{W}}{\rightarrow} \sum_{t=1}^{\infty} \sum_{k=0}^{\infty} \delta_{j_{t}\left(V_{t, k}, \ldots, V_{t, k-h}\right)}
$$

in $M_{\mathrm{p}}\left([-\infty, \infty]^{h+1} \backslash\{\mathbf{0}\}\right)$.

For $k=0, \ldots, m$, let $\boldsymbol{e}_{k}$ be the $k$ th standard unit vector in $\mathbb{R}^{m+1}$, i.e. the vector whose $k$ th component is 1 , the rest being 0 .

Proposition 3.1. Let $V_{t}=\left(V_{t}^{(0)}, \ldots, V_{t}^{(m)}\right)$, where $V_{t}^{(k)}=C_{t, k} Z_{t-k}, k=0, \ldots, m$. Under the assumptions of Theorem 3.1 we have

$$
\sum_{t=1}^{n} \delta_{a_{n}^{-1} \boldsymbol{V}_{t}} \stackrel{\mathrm{w}}{\rightarrow} \sum_{t=1}^{\infty} \sum_{k=0}^{m} \delta_{j_{t} V_{t, k} \boldsymbol{e}_{k}} .
$$


Proof. Let $E_{m}=[-\infty, \infty]^{m} \backslash\{\mathbf{0}\}$. We first show that

$$
\sum_{t=1}^{n} \delta_{\left(a_{n}^{-1}\left(Z_{t}, \ldots, Z_{t-m}\right), C_{t, 0}, \ldots, C_{t, m}\right)} \stackrel{\mathrm{W}}{\rightarrow} \sum_{t=1}^{\infty} \sum_{k=0}^{m} \delta_{\left(j_{t} e_{k}, V_{t, 0}, \ldots, V_{t, m}\right)}
$$

in $M_{\mathrm{p}}\left(E_{m+1} \times[-\infty, \infty]^{m+1}\right)$. Let

$$
\boldsymbol{A}_{n, t}^{(m)}=\left(a_{n}^{-1}\left(Z_{t}, \ldots, Z_{t-m}\right), C_{t, 0}, \ldots, C_{t, m}\right) .
$$

The random vectors $\boldsymbol{A}_{n, t}^{(m)}$ have the following properties.

- The sequence $\left\{\boldsymbol{A}_{n, t}^{(k)}, t \geq 1\right\}$ is stationary and strongly mixing, by (E).

- If $F_{m}$ is a distribution of $\left(C_{t, 0}, \ldots, C_{t, m}\right)$, then

$$
\begin{aligned}
& n \mathrm{P}\left(a_{n}^{-1}\left(Z_{t}, \ldots, Z_{t-m}\right) \in\left(\mathrm{d} z_{0}, \ldots, \mathrm{d} z_{m}\right),\left(C_{t, 0}, \ldots, C_{t, m}\right) \in\left(\mathrm{d} x_{0}, \ldots, \mathrm{d} x_{m}\right)\right) \\
& \quad \rightarrow \sum_{k=0}^{m} v\left(\mathrm{~d} z_{k}\right) \prod_{j \neq k} \delta_{0}\left(\mathrm{~d} z_{j}\right) F_{m}\left(\mathrm{~d} x_{0}, \ldots, \mathrm{d} x_{m}\right),
\end{aligned}
$$

by $(\mathrm{B})$ and $\left(\mathrm{C}^{\prime}\right)$.

- For all $g \in C_{K}^{+}\left(E_{m+1} \times[-\infty, \infty]^{m+1}\right)$,

$$
\lim _{m \rightarrow \infty} \limsup _{n \rightarrow \infty} n \sum_{t=2}^{[n / m]} \mathrm{E}\left[g\left(\boldsymbol{A}_{n, 1}^{(k)}\right) g\left(\boldsymbol{A}_{n, t}^{(k)}\right)\right]=0 .
$$

Indeed, let $B$ be a compact subset of $E_{1}$ and assume that $g$ has support contained in $B_{1}=(B \times[-\infty, \infty])^{m+1}$. By (A), we have

$$
\begin{aligned}
\limsup _{n \rightarrow \infty} n \sum_{t=2}^{[n / m]} \mathrm{E}\left[g\left(\boldsymbol{A}_{n, 1}^{(k)}\right) g\left(\boldsymbol{A}_{n, t}^{(k)}\right)\right] & \leq \limsup _{n \rightarrow \infty} \sum_{t=2}^{[n / m]} \mathrm{P}\left(a_{n}^{-1} Z_{1-k} \in B, a_{n}^{-1} Z_{t-k} \in B\right) \\
& =\limsup _{n \rightarrow \infty} \frac{n^{2}}{m} \mathrm{P}\left(a_{n}^{-1} Z \in B\right)^{2},
\end{aligned}
$$

which converges to 0 as $m \rightarrow \infty$, by (B).

Hence, (3.1) follows by Theorem 2.1 of [7]. Now let

$$
g_{i, m}\left(x_{0}, \ldots, x_{m}, u_{0}, \ldots, u_{m}\right)= \begin{cases}x_{i} u_{i} & \text { if } u_{i}<\infty \\ 0 & \text { otherwise }\end{cases}
$$

This induces a continuous mapping from $M_{\mathrm{p}}\left(E_{m+1} \times[-\infty, \infty]^{m+1}\right)$ into $[-\infty, \infty]$. Therefore, the result follows (see the proof of Proposition 3.2 of [8] for technical details).

Proof of Theorem 3.1. By Proposition 3.1 and an application of continuous mapping, we have

$$
\sum_{t=1}^{n} \delta_{a_{n}^{-1} \sum_{k=0}^{m} V_{t}^{(k)}} \stackrel{\mathrm{W}}{\rightarrow} \sum_{k=0}^{m} \sum_{t=1}^{\infty} \delta_{j_{t} V_{t, k}}
$$


Note that $X_{t}=\sum_{k=0}^{\infty} V_{t}^{(k)}$; it thus suffices to show that

$$
\lim _{m \rightarrow \infty} \limsup _{n \rightarrow \infty} \mathrm{P}\left(\left|\sum_{t=1}^{n} f\left(a_{n}^{-1} \sum_{k=0}^{m} V_{t}^{(k)}\right)-\sum_{t=1}^{n} f\left(a_{n}^{-1} X_{t}\right)\right|>\xi\right)=0
$$

for all $\xi>0$ and $f \in C_{K}^{+}\left(E_{1}\right)$; cf. [13, Proposition 4.27]. By Proposition 2.1, for each $\theta>0$ we have

$\lim _{m \rightarrow \infty} \limsup _{n \rightarrow \infty} n \mathrm{P}\left(\left|\sum_{k=0}^{m} V_{t}^{(k)}-X_{t}\right|>a_{n} \theta\right)=\lim _{m \rightarrow \infty} \limsup _{n \rightarrow \infty} n \mathrm{P}\left(\sum_{k=m+1}^{\infty} C_{t, k} Z_{t-k}>a_{n} \theta\right)=0$

and, hence, (3.2) follows.

Proof of Theorem 3.2. Assume that $h=1$ and note that

$$
\left(V_{t}^{(1)}, \ldots, V_{t}^{(m)}, V_{t-1}^{(0)}, \ldots, V_{t-1}^{(m-1)}\right)=\left(Z_{t-1}\left(C_{t, 1}, C_{t-1,0}\right), \ldots, Z_{t-m}\left(C_{t, m}, C_{t-1, m-1}\right)\right) .
$$

Thus, if

$$
\begin{gathered}
\boldsymbol{R}_{t}^{(m)}=\left(Z_{t-1}(1,1), \ldots, Z_{t-m}(1,1) ; C_{t, 1}, C_{t-1,0}, \ldots, C_{t, m}, C_{t-1, m-1}\right)=:\left(\boldsymbol{Z}_{t}^{(m)} ; \boldsymbol{D}_{t}^{(m)}\right), \\
z=\left(z_{1}, \ldots, z_{2 m}\right), \quad \text { and } \quad \boldsymbol{x}=\left(x_{1}, \ldots, x_{2 m}\right),
\end{gathered}
$$

then

$$
n \mathrm{P}\left(a_{n}^{-1} \boldsymbol{Z}_{t}^{(m)} \in \mathrm{d} \boldsymbol{z}, \boldsymbol{D}_{t}^{(m)} \in \mathrm{d} \boldsymbol{x}\right) \rightarrow \sum_{k=0}^{m-1} v\left(\mathrm{~d} z_{2 k+1}\right) \delta_{z_{2 k+1}}\left(\mathrm{~d} z_{2 k+2}\right) \prod_{j \neq 2 k+1,2 k+2} \delta_{0}\left(\mathrm{~d} z_{j}\right) G_{m}(\mathrm{~d} \boldsymbol{x}),
$$

where $G_{m}$ is a distribution of $\boldsymbol{D}_{t}^{(m)}$. We may conclude our proof using the same method as in the proof of Theorem 3.1, coupled with the proof of Theorem 3.4(ii) of [8].

Example 3.1. Consider the stochastic recurrence equation (1.3) and assume that $\left\{Y_{t}, t \in \mathbb{Z}\right\}$ is independent of $\left\{Z_{t}, t \in \mathbb{Z}\right\}$. Because $C_{t, k}=\prod_{i=0}^{k-1} Y_{t-i}$, it is easy to show that the vectors $\boldsymbol{A}_{t}=\left(C_{t, 0}, \ldots, C_{t, m}\right)$ form an $m$-dependent and, hence, strongly mixing sequence. Condition (E) is fulfilled. The random variables $V_{t, k}$ may be given in the form $V_{t, k}=\prod_{i=0}^{k-1} R_{t, i}$, where $R_{t, i}, t \geq 1, i \geq 1$, are i.i.d. with the same distribution as $Y$, with $V_{t, 0} \equiv 1$. Note, however, that in [11] the existence of a point process limit was established without the independence assumption.

\section{Applications}

\subsection{Partial sums}

Using Theorems 3.1 and 3.2 we may establish many limit results. For instance, assume that $\alpha \in(0,1)$. Then $X_{t}$ is regularly varying with index $\alpha \in(0,1)$. Define $S_{n}=\sum_{t=1}^{n} X_{t}$. By applying Theorem 3.1 of [4] to our Theorem 3.1, we have

$$
a_{n}^{-1} S_{n} \stackrel{\mathrm{w}}{\rightarrow} \sum_{t=1}^{\infty} \Gamma_{t}^{-1 / \alpha} A_{t}
$$

where $A_{t}=\sum_{k=0}^{\infty} V_{t, k}$ and $\Gamma_{t}, t \geq 1$, are points of a standard Poisson process. The limiting random variable is stable. 


\subsection{Self-normalized partial sums}

To establish results for partial sums we use normalization constants $a_{n}$ that depend on the tail of $Z$ and, thus, on $\alpha$. In practice $\alpha$ is very often unknown. Therefore, we consider the limit for self-normalized partial sums (cf. [3]). By considering two almost-surely continuous mappings acting on the same point process we can treat them as one almost-surely continuous mapping. Therefore, for $\alpha \in(0,1)$,

$$
\left(\max _{i=1, \ldots, n} a_{n}^{-1} X_{i}, a_{n}^{-1} \sum_{t=1}^{n} X_{t}\right) \stackrel{\mathrm{w}}{\rightarrow}\left(\max _{t \geq 1} \Gamma_{t}^{-1 / \alpha} A_{t}, \sum_{t=1}^{\infty} \Gamma_{t}^{-1 / \alpha} A_{t}\right)
$$

and, hence,

$$
\sum_{t=1}^{n} \frac{X_{t}}{\max \left\{X_{1}, \ldots, X_{n}\right\}} \stackrel{\mathrm{w}}{\rightarrow} \sum_{t=1}^{\infty} \frac{\Gamma_{t}^{-1 / \alpha} A_{t}}{\max _{t \geq 1} \Gamma_{t}^{-1 / \alpha} A_{t}} .
$$

We can observe another difference between moving average processes with random coefficients and the $\mathrm{MA}(\infty)$ model. Indeed, the limiting distribution in the random-coefficient model depends on the behavior of the sequence $\left\{A_{t}\right\}$, which in turns depends on the distributions of the $C_{t, k}$. In the latter case, bearing in mind that $A_{t}=\sum_{k=0}^{\infty} c_{k}$ does not depend on $t$, we have

$$
\sum_{t=1}^{n} \frac{X_{t}}{\max \left\{X_{1}, \ldots, X_{n}\right\}} \stackrel{\mathrm{w}}{\rightarrow} \sum_{t=1}^{\infty}\left(\frac{\Gamma_{1}}{\Gamma_{t}}\right)^{1 / \alpha},
$$

and the limit does not depend on the parameters $c_{k}$.

\subsection{Checking linearity}

Since the limiting behaviors of moving average processes with random coefficients and standard MA $(\infty)$ processes are different, we need statistical tests to detect possible nonlinearities. For infinite variance, this problem was studied in [15]. The approach taken there is based on the behavior of sample autocorrelation functions.

We propose a simple rule to detect nonlinearities in the AR(1) model. Assume that, based on observations $\left\{X_{t}, t=1, \ldots, n\right\}$, we want to check if these data come from the stochastic recurrence model (1.3) or from the AR(1) model. To do this assume that a distribution, $G$, of $Y$ has support $[a, b], a>0, b \leq \infty$. On the one hand, by Theorem 3.2 we may conclude (cf. [14]) that

$$
\sum_{t=2}^{n} \delta_{X_{t} / X_{t-1}} \stackrel{\mathrm{w}}{\rightarrow} \sum_{k=1}^{\infty} \sum_{t=1}^{\infty} \delta_{R_{t, k}}
$$

and, hence,

$$
\hat{a}_{n}:=\bigwedge_{t=2}^{n} \frac{X_{t}}{X_{t-1}} \stackrel{\mathrm{w}}{\rightarrow} a, \quad \hat{b}_{n}:=\bigvee_{t=2}^{n} \frac{X_{t}}{X_{t-1}} \stackrel{\mathrm{w}}{\rightarrow} b .
$$

On the other hand, for the $\operatorname{AR}(1)$ model $X_{t}=\rho X_{t-1}+Z_{t},|\rho|<1$, we have

$$
\bigwedge_{t=2}^{n} \frac{X_{t}}{X_{t-1}}-\bigvee_{t=2}^{n} \frac{X_{t}}{X_{t-1}} \stackrel{\mathrm{w}}{\rightarrow} \rho-\rho=0
$$

Thus, $\left|\hat{a}_{n}-\hat{b}_{n}\right|$ being small indicates that the model is linear, and we may then proceed with a test proposed in [15]. 
It should be pointed out that this approach compares only the stochastic recurrence model with the AR(1) model. Indeed, assume that the observations come from an $\mathrm{AR}(2)$ process. It can be represented as the $\mathrm{MA}(\infty)$ process $(1.1)$, in which case

$$
\bigwedge_{t=2}^{n} \frac{X_{t}}{X_{t-1}}-\bigvee_{t=2}^{n} \frac{X_{t}}{X_{t-1}} \stackrel{\mathrm{w}}{\rightarrow} \bigwedge_{k=1}^{n} \frac{c_{k}}{c_{k-1}}-\bigvee_{k=1}^{n} \frac{c_{k}}{c_{k-1}}
$$

Thus, in general, the limit need not be 0 .

\section{Acknowledgements}

The author would like to thank the referee and Professor André Dabrowski for many helpful comments that improved the paper.

\section{References}

[1] Bougerol, P. And Picard, N. (1992). Strict stationarity of generalized autoregressive processes. Ann. Prob. 20, 1714-1730.

[2] Breiman, L. (1965). On some limit theorems similar to the arc-sin law. Theory Prob. Appl. 10, 351-360.

[3] Dabrowski, A. R., Dehling, H. G., Mikosch, T. and Sharipov, O. (2002). Poisson limits for $U$-statistics. Stoch. Process. Appl. 99, 137-157.

[4] Davis, R. A. And Hsing, T. (1995). Point process and partial sum convergence for weakly dependent random variables with infinite variance. Ann. Prob. 23, 879-917.

[5] Davis, R. A. And Mikosch, T. (1998). The sample autocorrelations of heavy-tailed processes with applications to ARCH. Ann. Statist. 26, 2049-2080.

[6] Davis, R. A. AND Resnick, S. I. (1985). Limit theory for moving averages of random variables with regularly varying tail probabilities. Ann. Prob. 13, 179-195.

[7] Davis, R. A. AND Resnick, S. I. (1988). Extremes of moving averages of random variables from the domain of attraction of the double exponential distribution. Stoch. Process. Appl. 30, 41-68.

[8] Davis, R. A. And Resnick, S. I. (1996). Limit theory for bilinear processes with heavy-tailed noise. Ann. Appl. Prob. 6, 1191-1210.

[9] Embrechts, P., Klüppelberg, C. And Mikosch, T. (1987). Modelling Extremal Events for Insurance and Finance (Appl. Math. (New York) 33). Springer, Berlin.

[10] Goldie, C. M. (1991). Implicit renewal theory and tails of solutions of random equations. Ann. Appl. Prob. 1, $126-166$.

[11] Konstantinides, D. G. and Mikosch, T. (2005). Large deviations and ruin probabilities for solutions to stochastic recurrence equations with heavy-tailed innovations. Ann. Prob. 33, 1992-2035.

[12] Мiкоsсн, T. (2004). Modelling dependence and tails of financial time series. In Extreme Values in Finance, Telecommunications, and the Environment, eds B. Finkenstädt and H. Rootzén, Chapman and Hall, Boca Raton, FL, pp. 185-286.

[13] ReSnICK, S. I. (1987). Extreme Values, Regular Variation, and Point Processes. Springer, New York.

[14] ReSnick, S. I. (1998). Why non-linearities can ruin the heavy-tailed modeler's day. In A Practical Guide to Heavy Tails, eds R. J. Adler, R. E. Feldman and M. S. Taqqu, Birkhäuser, Boston, MA, pp. 219-239.

[15] Resnick, S. I. AND VAN DEN Berg, E. (2000). A test for nonlinearity of time series with infinite variance. Extremes 3, 145-172.

[16] Resnick, S. I. AND Willekens, E. (1991). Moving averages with random coefficients and random coefficient autoregressive models. Commun. Statist. Stoch. Models 7, 511-525. 